\title{
CSMJ
}

\section{Evaluation of Survival of Patients Who Underwent Decompressive Craniectomy: Clinical Series}

\author{
(D) Erkan Kutlu Ekiz, (D) Ozan Barut, (D) Ozan Haşimoğlu, (D) Yusuf Kılıç \\ University of Health Sciences Turkey, Başakşehir Çam and Sakura City Hospital, Clinic of Neurosurgery, İstanbul, \\ Turkey
}

What is known on this subject?

Rapid emergency surgical treatment is important in patients with intracranial hematoma.

\section{What this study adds?}

When the Glasgow Coma scale score is 10 and below, rapid surgical treatment is not important.

\section{ABSTRACT}

Objective: This study aimed to examine the parameters thought to reduce the mortality of patients with epidural-subdural hemorrhage, basal ganglia hemorrhage, edema with compression effect due to intracerebral ischemic infarction, and hemorrhage from infarcts and to find significant relationships accordingly.

Material and Methods: The demographic and clinical characteristics of patients, pre-operative Glasgow Coma scale (GCS), duration of the operation after the development of the first event, length of stay in intensive care units (ICUs), infection and antibiotic therapy rates developed during their hospitalization, and long-term follow-up were recorded. The survival of the patients were compared statistically.

Results: In 38 patients with GCS less than 10 points, pre-operative GCS, length of stay in ICUs, duration of mechanical ventilator support, infection, and need for antibiotic therapy were examined. All patients were divided into four groups; subdural-epidural hematoma, intracerebral hematoma, intracerebral ischemic infarction, and post-infarction hemorrhage groups. The relationship between their data and mortality were studied. The pre-operative GCS scores in the four groups were $6.16,6.73,7.13$, and 6.28 , respectively. The pre-operative GCS in these four dead groups were $5,6.6,7$, and 6 , respectively. There was no difference between the variables and mortality.

Conclusion: No correlation was found between all clinical data and survival rates. The benefits of an early surgery shown in previous studies were not associated with mortality in this study. Studies with larger case series are needed for more significant relationships.

Keywords: Cerebral ischemic stroke, decompressive craniectomy, epidural hemorrhage, intracerebral hemorrhage, mortality, subdural hemorrhage

Address for Correspondence: Erkan Kutlu Ekiz MD, University of Health Sciences Turkey, Başakşehir Çam and Sakura City Hospital, Clinic of Neurosurgery, İstanbul, Turkey

Phone: +90 5326717131 E-mail: ekutlue@hotmail.com ORCID ID: orcid.org/0000-0002-5661-8892 Received: 27.01.2021 Accepted: 09.03.2021

(c) Copyright 2021 by the Cam \& Sakura Medical Journal published by Galenos Publishing House. 


\section{Introduction}

Decompressive craniectomy (DC) aims to reduce intracranial pressure, which is increased due to epiduralsubdural hematoma, intracerebral hematoma, and ischemia. The effects of cell loss due to increased intracranial pressure and low cerebral perfusion caused by blood and inflammatory mediators on mortality and morbidity are already known (1). DC reduces intracerebral pressure (ICP), but its effect on mortality and morbidity is uncertain (2). However, DC is the most commonly used surgical treatment for increased ICP after trauma, hemorrhage, or ischemia. This study aimed to investigate the effects of demographic and clinical features on the survival of patients who underwent DC for traumatic or non-traumatic reasons.

\section{Material and Methods}

Patients who underwent DC in our clinic between 2016 and 2017 due to traumatic intracerebral hematoma, subdural/ epidural hematoma, hypertensive basal ganglia hemorrhage, and post-acute ischemic/hemorrhagic infarct edema and hematoma were included in the study. Frontoparietal DC was performed on the shifted side in all patients. The bone was placed in a subcutaneous pocket in the abdomen. The bone was placed back after six months of healing. Demographic and clinical features, Glasgow Coma scale (GCS) scores, the time between the incident and surgery, period of stay in intensive care units (ICUs), infections, and antibiotic treatments were recorded. These data were compared statistically with the survival of the patients retrospectively. These data were obtained from the Bakırköy Prof. Dr. Mazhar Osman Training and Research Hospital for Psychiatric, Neurologic, and Neurosurgical Diseases database.

\section{Exclusion Criteria}

Patients under 12 years of age and those who underwent DC for reasons other than traumatic intracerebral hematoma, subdural/epidural hematoma, hypertensive cerebral hemorrhage, and acute ischemic/hemorrhagic infarction were excluded in this study.

\section{Statistical Analysis}

Physiological measurements within groups were analyzed by the Student's t-test for paired data and Pearson's chisquare test for independent samples (two-tailed) using a statistical software (SPSS version 10.0, Chicago, IL). $\mathrm{P}<0.05$ was considered statistically signficant.

\section{Results}

Thirty-eight patients, 10 female and 28 male, were included in this study. Eight patients had hypertensive intracerebral hematoma, seven had post-infarction hematoma, 11 had extensive ischemic infarction, and 12 had subdural/traumatic hematoma. The mean GCS score of all patients on admission to the operation was 6.73. The mean GCS score of patients who died during follow-up on admission was 6.00. Moreover, $74 \%$ of the patients were discharged with recovery.

The mean GCS score of patients with ischemic infarction who underwent DC was 7.13. The mean operation time was $66.76 \mathrm{~h}$. The mean operation time of patients who died was $38.23 \mathrm{~h}$. Early surgery was not found to be significant in this group in terms of mortality. The mean ICU follow-up was 31.5 days. The mean follow-up time with a mechanical ventilator was 19.09 days. The mean GCS score of patients who died was 7. The mean ICU follow-up was three days, and the mean follow-up time with a mechanical ventilator was 3.3 days. No significant difference was found between the variables and survivalm rates of these groups.

In patients with post-infarction hematoma, the average GCS score was 6.28. The mean ICU follow-up was 11.4 days, and the mean follow-up with a mechanical ventilator was 4.8 days. The mean admission GCS score of patients who died was 6 , the mean number of ICU follow-up duration was 9 , and the mean follow-up with a mechanical ventilator was 6 days. No significant difference was found between the variables and survival rates of these groups.

In patients with subdural/traumatic hematoma, the mean GCS score of all patients was 6.16. The mean follow-up period was 35.35 days, and the mean follow-up with a mechanical ventilator was 8.4 days. Of the six patients who died, the mean GCS score was 5.00, the mean ICU follow-up duration was 48.50 , and the mean follow-up with a mechanical ventilator was 7.4 days. No significant difference was found between the variables and survival rates of these groups.

Eight patients with hypertensive intracerebral hematoma were included in this study. The mean GCS score of all patients with intracerebral hematoma was 6.73. The mean ICU followup time was 18.74 days, and the mean mechanical ventilator follow-up time was 11.24 days. The GCS score of deceased patients was 9. The number of ICU follow-up days was 41, and the follow-up period with a mechanical ventilator was 35 days. No significant difference was found between the variables and survival rates of these groups.

The number of patients who received prophylactic antibiotic therapy was 34 , while 25 patients developed an 
infection and received systemic antibiotics. Six of these patients died. There was no significant difference between the infection and survival All data are summarized in Table 1.

\section{Discussion}

This DC case series was reviewed retrospectively from a group of patients with long-term follow-up period. Patients who underwent DC for subdural hematoma, intracerebral hematoma, post-infarction hematoma, and ischemic infarction were included in this study. The relationship between the clinical features of these patients and their mortality was compared statistically. No correlation was found between their mortality values, demographic characteristics, clinical features, initial GCS scores, duration of operation after the development of the first event, length of stay in ICUs, and infection. In this study, all patients who underwent DC had a GCS score of $<10$. The GCS score at the time of entry to the operation did not contribute to mortality. In STICH and STICH II trials, which allow a multicenter meta-analysis, the survival and clinical outcome scores of the patients who underwent surgical intervention when the GCS scores $\geq 12$ increased $(3,4)$. The subject of rapid surgery was included in this study. However, since there was no patient with a GSC score $>10$ points, this study produced a different result from that in other studies. These studies suggest that the rapid surgical intervention, which has a positive effect on mortality, is not suitable for patients with a GCS score of $\leq 10$.

The aim of the treatment and follow-up in patients with acute subdural hematoma and intracerebral hematoma was to reduce brain damage due to bleeding, to stop the progression of rapid neurological regression, and to reduce the elevated intracranial pressure and mass effect. However, the postoperative clinical results of patients with subdural and intracerebral hematoma are not satisfactory. Nevertheless, DC is considered superior to the maximum conservative treatment (5).

The most common causes of intracerebral hematomas are hypertensive, vascular, and hemorrhages secondary to amyloid deposition (5). Although the use of a minimally invasive hematoma drainage treatment for hypertensive hematomas can be observed in the basal ganglia, which generally causes low GCS scores, serious neurological deficits, and very poor prognosis, DC is still the most used method worldwide (6). In intracerebral hematomas, perihematomal edema increases by $75 \%$ in the first $24 \mathrm{~h}$ (6). One of the reasons why DC is the most preferred method for the surgical treatment of intracerebral hematoma is because of its effectiveness in eliminating the compression effect regardless of the location of the hematoma (6). Despite this, there is still no clear consensus on regulations that will increase their survival. No statistically significant survival parameter was found in this study.

DC is also widely used in diffuse intracerebral edema that develops after an ischemic stroke that causes a compression effect. An impaired cerebral perfusion and cerebral oxygenation due to edema increases the amount of edema, creating a vicious circle (7). In previous studies, in patients who needed DC after an ischemic stroke, it has been reported

Table 1. Patient who had decompressive craniectomy clinica follow-up data

\begin{tabular}{|c|c|c|c|c|c|c|c|}
\hline & & & & All patients & & & \\
\hline & Female/male & Age & Initial GCS & $\begin{array}{l}\text { ICU follow-up } \\
\text { days }\end{array}$ & $\begin{array}{l}\text { Mechanical ventilator } \\
\text { follow-up days }\end{array}$ & Infection & $\begin{array}{l}\text { Antibiotic } \\
\text { treatment }\end{array}$ \\
\hline Infarction & $5 / 13$ & 61.6 & 6.94 & 23.7 & 13.5 & $12 / 18$ & $14 / 18$ \\
\hline & & & Ex patient & & & & \\
\hline & Female/male & Age & Initial GCS & $\begin{array}{l}\text { ICU follow-up } \\
\text { days }\end{array}$ & $\begin{array}{l}\text { Mechanical ventilator } \\
\text { follow-up days }\end{array}$ & Infection & $\begin{array}{l}\text { Antibiotic } \\
\text { treatment }\end{array}$ \\
\hline $\begin{array}{l}\text { Subdural } \\
\text { hematoma }\end{array}$ & $3 / 3$ & 65.5 & 5 & 48.5 & 7.4 & $2 / 6$ & $4 / 6$ \\
\hline Post-infarction ICH & $1 / 3$ & 63.5 & 6.0 & 9.0 & 6.0 & $2 / 4$ & $2 / 4$ \\
\hline
\end{tabular}


that an early planning for surgery is effective in improving neurological deficits and preserving other brain functions (8). In our study, no data were found to show the positive effects of an early surgery on mortality.

In this study, the ICU period, infection, need for antibiotic therapy, and duration of mechanical ventilator use were examined. Most of the patients included in this study needed mechanical ventilators. In addition, they received an antibiotic therapy due to infections that developed in various locations in the body outside the surgical area. However, no significant difference was found between these variables. It was also shown that the rate of infection after DC increases the risk of mortality (9).

\section{Study Limitations}

The main limitation of the study was the small number of patients, which was less than the population prevalence, and the short examination time for the follow-up group. In addition, because all patients were under different surgeons and neurologists, the timing of surgery may have been decided subjectively. A significant level of evidence on this subject has not yet been reached (10). In addition, in this study, the operation times were not recorded, and the patients were operated on quickly after the event occurred, except for the ischemic infarct group. To improve the survival after DC, it would be beneficial to work with more standardized and larger patient groups.

\section{Conclusion}

In this study, no correlation was found the between the clinical features and mortality of the patients who underwent DC. The relationships between these variables can be shown more clearly in future large case series.

\section{Ethics}

Ethics Committee Approval: Approval was obtained from the Başakşehir Çam and Sakura Hospital Clinical Research Ethics Committee (protocol no: 2021-182).

Informed Consent: Retrospective study.

Peer-review: Externally peer-reviewed.

\section{Authorship Contributions}

Surgical and Medical Practices: E.K.E., O.H., O.B., Y.K., Concept: E.K.E., O.H., Design: E.K.E., O.H., Data Collection or Processing: O.B., Y.K., Analysis or Interpretation: O.B., Y.K., Literature Search: E.K.E., O.H., Writing: E.K.E., O.H.

Conflict of Interest: No conflict of interest was declared by the authors.

Financial Disclosure: The authors declared that this study received no financial support.

\section{REFERENCES}

1. de Jonge JC, Takx RAP, Kauw F, de Jong PA, Dankbaar JW, van der Worp HB. Signs of pulmonary infection on admission chest computed tomography are associated with pneumonia or death in patients with acute stroke. Stroke 2020;51:1690-1695.

2. Hutchinson PJ, Kolias AG, Timofeev IS, et al. Trial of decompressive craniectomy for traumatic intracranial hypertension. N Engl J Med 2016;375:1119-1130.

3. Mendelow AD, Gregson BA, Fernandes HM, et al. Early surgery versus initial conservative treatment in patients with spontaneous supratentorial intracerebral haematomas in the International Surgical Trial in Intracerebral Haemorrhage (STICH): a randomised trial. Lancet 2005;365:387-397.

4. Kolias AG, Kirkpatrick PJ, Hutchinson PJ. Decompressive craniectomy: past, present and future. Nat Rev Neurol 2013;9:405-415.

5. Lok J, Leung W, Murphy S, Butler W, Noviski N, Lo EH. Intracranial hemorrhage: mechanisms of secondary brain injury. Acta Neurochir Suppl 2011;111:63-69.

6. Xiao K, Chu H, Chen H, Zhong Y, Zhong L, Tang Y. Optimal time window for minimally invasive surgery in treating spontaneous intracerebral hemorrhage in the basal ganglia region: a multicenter and retrospective study. Br J Neurosurg 2020;8:1-5.

7. Kolias AG, Adams H, Timofeev I, et al. Decompressive craniectomy following traumatic brain injury: developing the evidence base. $\mathrm{Br}$ J Neurosurg 2016;30:246-250

8. Lanzino G. Decompressive craniectomy for acute stroke: early is better. Journal of Neurosurgery 2008;109:285-2853.

9. Miller JD, Becker DP, Ward JD, Sullivan HG, Adams WE, Rosner MJ. Significance of intracranial hypertension in severe head injury. J Neurosurg 1977; 47:503-516

10. Powers WJ, Derdeyn CP, Biller J, et al. 2015 American Heart Association/American Stroke Association Focused Update of the 2013 Guidelines for the Early Management of Patients with Acute Ischemic Stroke Regarding Endovascular Treatment: A Guideline for Healthcare Professionals From the American Heart Association/ American Stroke Association. Stroke 2015;46:3020-3035. 